\title{
Realistic deformable 3D numeric phantom for transcutaneous ultrasound
}

\author{
Fernando Mitsuyama Cardoso ${ }^{1 *}$, Matheus Cardoso Moraes $^{2}$, Sergio Shiguemi Furuie ${ }^{1}$ \\ ${ }^{1}$ School of Engineering, University of São Paulo, São Paulo, SP, Brazil. \\ ${ }^{2}$ Institute of Science and Technology, Federal University of São Paulo, São José dos Campos, SP, Brazil.
}

\begin{abstract}
Introduction: Numerical phantoms are important tools to design, calibrate and evaluate several methods in various image-processing applications, such as echocardiography and mammography. We present a framework for creating ultrasound numerical deformable phantoms based on Finite Element Method (FEM), Linear Isomorphism and Field II. The proposed method considers that the scatterers map is a property of the tissue; therefore, the scatterers should move according to the tissue strain. Methods: First, a volume representing the target tissue is loaded. Second, parameter values, such as Young's Modulus, scatterers density, attenuation and scattering amplitudes are inserted for each different regions of the phantom. Then, other parameters related to the ultrasound equipment, such as ultrasound frequency and number of transducer elements, are also defined in order to perform the ultrasound acquisition using Field II. Third, the size and position of the transducer and the pressures that are applied against the tissue are defined. Subsequently, FEM is executed and deformation is computed. Next, 3D linear isomorphism is performed to displace the scatterers according to the deformation. Finally, Field II is carried out to generate the non-deformed and deformed ultrasound data. Results: The framework is evaluated by comparing strain values obtained the numerical simulation and from the physical phantom from CIRS. The mean difference between both phantoms is lesser than 10\%. Conclusion: The acoustic and deformation outcomes are similar to those obtained using a physical phantom. This framework led to a tool, which is available online and free of charges for educational and research purposes.
\end{abstract}

Keywords Ultrasound images, Simulation, Numerical phantoms, Elasticity, Linear isomorphism.

\section{Introduction}

Diagnostic Ultrasonography (US) has been widely used for more than 50 years and is an established medical imaging technique based on reflected ultrasound waves, which provides tomographic view of subcutaneous structures, important information for diagnosis, therapy and evaluations. However, in some cases, US images alone may not provide sufficient information to objectively and precisely qualify a lesion composition and dangerousness. Therefore, although it has long been used, several researches continuously investigate new image processing tools and methods to improve US diagnostic capability or to create new applications (Ragazzoni et al., 2012), for instance ultrasound elastography and segmentation.

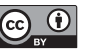

This is an Open Access article distributed under the terms of the Creative Commons Attribution License, which permits unrestricted use, distribution, and reproduction in any medium, provided the original work is properly cited.

How to cite this article: Cardoso FM, Moraes MC, Furuie SS. Realistic deformable 3D numeric phantom for transcutaneous ultrasound. Res Biomed Eng. 2017; 33(1):1-10. DOI: 10.1590/2446-4740.0056

*Corresponding author: School of Engineering, University of São Paulo - USP, Av. Prof. Luciano Gualberto, Travessa 3, 158, sala D2-06, CEP 05508-970, São Paulo, SP, Brazil.

E-mail: fernando.okara@gmail.com

Received: 21 July 2016 / Accepted: 05 February 2017
Elastography is an image processing technique in which the mechanical property of tissues is displayed in a strain or elasticity map, which may be produced from images of the target region acquired under different pressures (Céspedes et al., 1993; Doyley, 2012; Kallel and Ophir, 1997; Loree et al., 1994; Ophir et al., 1996). Segmentation consists in delineating or highlighting related objects in an image, providing detailed and objective structural information (Kurnaz et al., 2007).

Physical and Numerical Phantoms are very important tools in many algorithm-development stages of image processing applications (Cardoso et al., 2012; Culjat et al., 2010; Hoskins, 2008; King et al., 2011). Due to the flexibility of controlling parameters and desired features, they are used to calibrate and to evaluate the performance of algorithms; hence, valuable for the development of image systems and techniques, such as filtering (Latifoğlu, 2013), segmentation (Kurnaz et al., 2007) and speckle tracking. In (Culjat et al., 2010) a review on tissue-mimicking physical phantoms for US imaging is described. The physical phantoms may be based on different materials such as condensed milk (Bude and Adler, 1995), agar (Browne et al., 2003) and polyvinyl alcohol cryogel (Liang et al., 2008). Numerical phantoms can be found in (Baldewsing et al., 2004; Bhatti and Sridhar-Keralapura, 2012; Le Floc'h et al., 2009; 
Maurice et al., 2004). Usually COMSOL Multiphysics software (Structural Mechanics Module, version 3.3, COMSOL, France) and ANSYS $5.7^{\circledR}$ software (Ansys, Inc., Cannonsburg, PA) are utilized to compute structural deformations, and MATLAB ${ }^{\circledR}$ (MathWorks, Inc., Natick, MA, USA) with Field II (Jensen and Nikolov, 2000; Jensen and Svendsen, 1992) for Ultrasound simulation.

In order to continue improving algorithm performance, creating alternative methods and achieving new breakthroughs, medical images with desirable features are required. Usually, investigators should have access to three sources of images: clinical, physical phantom and numerical phantom. Real US images are not always available for researches, due to ethics protocols, facilities and difficulties in collaboration policies; and, when they are available, obtaining images with desired features for specific investigation is another difficult obstacle, since parameters and desired features cannot be controlled. Constructing physical phantoms for elasticity purposes may also be a complex task, because selecting corresponding materials to replace the mechanical properties of tissues is difficult (Culjat et al., 2010). In addition, physical phantoms also require US equipment, so that the resulting images can be produced; thus, physical phantoms also require access to facilities or collaborations. Yet, due to computational advancements and techniques, numerical phantoms are becoming more realistic and complete, permitting researchers to execute numerous investigations with controlled and flexible parameters and desired features adjustments (Culjat et al., 2010). Consequently, they are a feasible and practical choice for creating, calibrating and evaluating new methodologies.

Numerical phantoms can be found in prior studies; however, they are not fully covered, as they are not the main topic, but an auxiliary tool for a main investigation. Therefore, approaches fully dedicated to creating of realistically deformable numerical phantoms are important to help investigators to achieve new breakthroughs in US image applications. Consequently, the present approach aims to describe a framework to realistically create deformable numerical phantoms for US applications. In Cardoso et al. (2012), intravascular ultrasound images may be simulated using planar numeric phantom with different intraluminal pressure.

This work presents a detailed framework to produce ultrasound images for transcutaneous applications from a phantom that is able to deform according to the pressure of the transducer surface. In order to simulate the ultrasound acquisition during a procedure with strain in a more realistic fashion, the proposed method consistently displaces the scatterers according to the tissue deformation. This article covers the phantom generation from 3D FEM (Finite Elements Method) to speckle noise simulation. The methodology was embedded in a Toolbox for MATLAB ${ }^{\circledR}$; the tool is available online at as citationware license type and can be used free of charge for research and educational purposes.

\section{Methods}

The proposed framework relies on FEM (Kattan, 2008; Reddy 2006; Zienkiewicz et al., 2010a; 2010b), linear isomorphism (Cardoso et al., 2012) and Field II (Jensen and Nikolov, 2000; Jensen and Svendsen, 1992) to perform realistic deformation, scatterers displacement and transcutaneous ultrasound simulation, respectively, in 3D phantoms. The deformation is performed according to the Young Modulus of each structure, transducer position and applied pressure. The resulting US data is generated by Field II, considering the acoustic parameters of each structure, for instance attenuation and scatterers density.

The material, equipment and software used during development and evaluation of UltraSSim were the elasticity phantom (QA Model 049A from CIRS, Norfolk, VA, USA), ultrasound system (SonixTouch from Ultrasonix, British Columbia, Canada), a personal computer (Intel Core i5, $2.53 \mathrm{GHz}, 4 \mathrm{~GB}$ of RAM, with Windows 764 bits), MATLAB ${ }^{\circledR}$ software (MathWorks, Inc., Natick, MA, USA), iso2mesh Toolbox (Fang and Boas, 2009) and Field II (Jensen and Nikolov, 2000; Jensen and Svendsen, 1992).

The evaluation was carried out by calculating the accuracy of structural deformations provided by FEM in the generated phantoms. The resulting strain of the numerical US phantoms were compared with the outcomes from the physical phantom.

The methodology has three main blocks: Numerical modelling, Volume deformation, and US image generation. In Numerical modelling, a 3D volume representing the anatomical subcutaneous features is constructed and loaded (Figure 1a). Subsequently, the different volume structures, corresponding to different tissues of subcutaneous areas, are identified; then, mechanical and acoustic properties are inserted in order to be used by FEM and Field II, respectively. The second section, Volume deformation, uses FEM for the mechanical simulation, where the Young's Modulus for each region, boundary condition, size, position and increment of pressure produced by the transducer are entered. Then, the finite element mesh $(\chi)$ is generated using iso2mesh (Fang and Boas 2009) and, with the parameters assigned, the deformed mesh is computed $\left(\chi_{d}\right)$ (Figures 1b) (Cardoso et al., 2011; Culjat et al., 2010; Kattan, 2008; Reddy, 2006; Zienkiewicz et al., 2010a; 2010b). Finally, in the US image generation block, the acoustic and strain information provided by the previous blocks is utilized to displace the scatterers through linear isomorphism. Next, Field II is performed to include the speckle noise and 


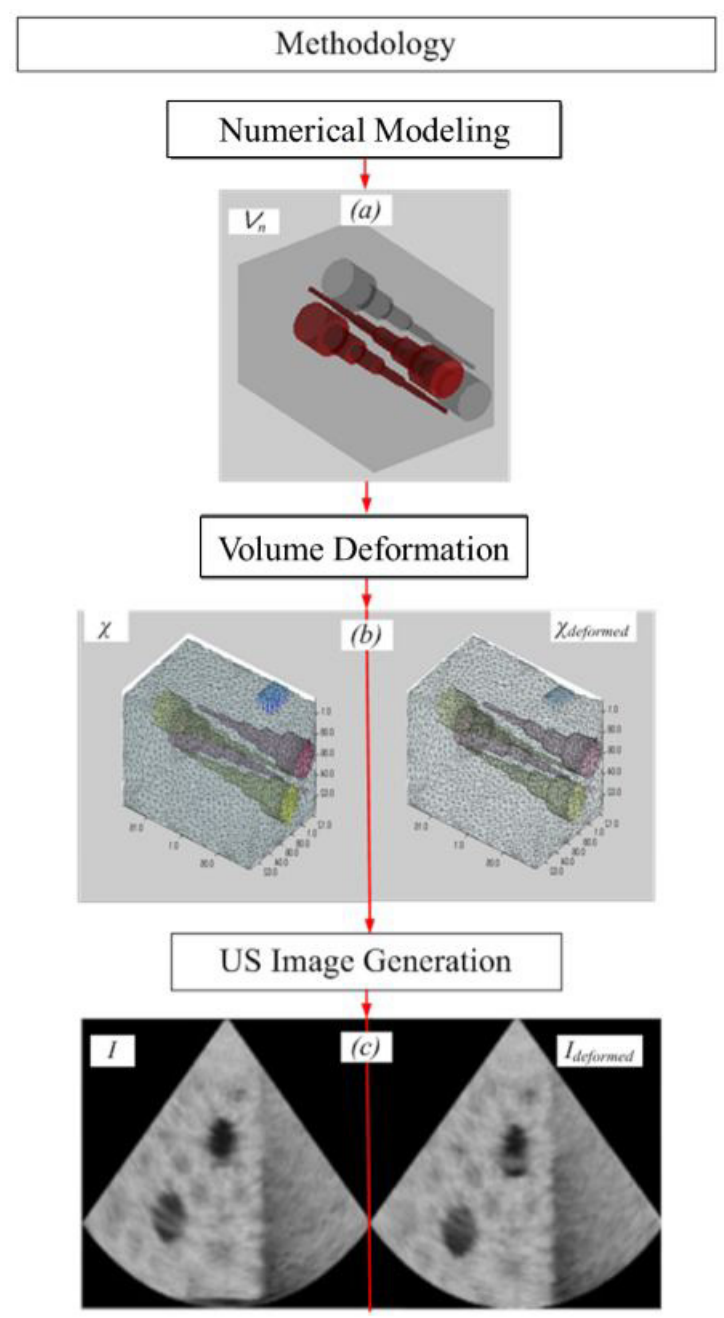

Figure 1. Methodology sequence to obtain the numerically simulated ultrasound images. First, a model representing the target tissue is created. Then, the tissue deformation due the pressure applied by the transducer is simulated through Finite Elements Method and the scatterers are displaced accordingly. Finally, the speckle noise is included using Field II and B-mode images are generated.

generate the noisy non-deformed $(I)$, and noisy deformed $\left(I_{d v}\right)$ US images (Figures 1c) (Anton and Rorres, 2005; Cardoso et al., 2011; 2012; Jensen and Nikolov, 2000; Jensen and Svendsen, 1992).

\section{Numerical modelling}

As previously described, a transcutaneous US has different applications; hence, the volumetric structure has to be designed to represent the regions of a determined application, for instance cyst, cancer, lipidic, fibrous or calcified tissues, etc. Therefore, the morphological, mechanical and acoustic properties of the volumetric structure must produce US images with corresponding deformation and echoic values in the slice.
The morphology of the volume may be built by combining well-known geometric functions, such as cylinder, spheres and squares, or may be imported from other sources. In this work, the input that governs the morphology of the volumetric phantom was a 3D matrix. In order to distinguish regions, to insert different mechanical and acoustic properties in the different areas, the voxels of each structure is assigned a different value, as illustrated in Figure 2a. If there are two disjoint groups of voxels with the same value, the algorithm should recognize them as being made of the same material. Although we propose creating of volumetric phantoms (3D), a planar view (2D) with low resolution is represented in Figure 2a-c for clarity.

The acoustic and mechanical properties can be regionally inserted by the investigator according to the application. In this work, we created a numerical phantom (Figure 2d), that simulates the physical elasticity QA phantom Model 049A from CIRS (Norfolk, VA, US), which is a physical phantom box that contains a background and isoechoic stepped cylinders representing 4 lesions with different mechanical properties. The Young Modulus of each tissue correspond to $25 \pm 6 \mathrm{kPa}, 8 \pm 3 \mathrm{kPa}, 14 \pm 4 \mathrm{kPa}, 45 \pm 8 \mathrm{kPa}$, $80 \pm 12 \mathrm{kPa}$, for the background, and lesions I, II, III, IV, respectively. The phantom width, length and height were $120.0 \mathrm{~mm}, 180.0 \mathrm{~mm}$ and $95.0 \mathrm{~mm}$, respectively. Table 1 summarizes the values of scatterer density and amplitude, attenuation and Young's Modulus of different regions are inserted for each region of the phantom. Although the background and inclusions of the physical phantom are isoechoic, we here considered the inclusions to be hypoechoic in relation to the background. Therefore, it is possible to visualize the inclusions in B-mode images.

In Figure 2d, the transducer surface is represented by a dark blue rectangle. The transducer characteristics are also inserted: features such as position, size and applied pressure, number of active elements at emission and reception, ultrasound center frequency, type of emission - linear or angular direction. In this work, we defined the transducer parameters as shown in Table 2.

\section{Volume deformation}

Since we are interested in simulating ultrasound procedures in which the insonified tissue is under strain, the numeric phantom, along with its structures, must be able do deform realistically. Therefore, in order to perform the simulation of the tissue, a polygonal mesh is generated according to the volume morphology (Figure 3a). Then, the mesh deformation relies on FEM to represent the mechanical features of the volume and its structures, together with the simulation of the dynamics of structural deformation with locally applied pressure (Figure $3 b$ ). 
\begin{tabular}{|l|l|l|l|l|l|l|l|l|l|l|l|l|l|l|l|l|l|l|l|l|l|l|}
\hline 1 & 1 & 1 & 1 & 1 & 1 & 1 & 1 & 1 & 1 & 1 & 1 & 1 & 1 & 1 & 1 & 1 & 1 & 1 & 1 & 1 & 1 & 1 \\
\hline
\end{tabular} \begin{tabular}{llll|l|l|l|l|l|l|l|l|l|l|l|l|l|l|l|l|l|l|l|l|}
1 & 1 & 1 & 1 & 1 & 1 & 1 & 1 & 1 & 1 & 1 & 1 & 1 & 1 & 1 & 1 & 1 & 1 & 1 & 1 & 1 & 1 & 1 \\
\hline
\end{tabular}

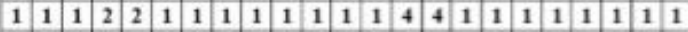
\begin{tabular}{llllllllllllllllllll|l|l|l|l|l|l|l|}
1 & 1 & 2 & 2 & 2 & 2 & 1 & 1 & 2 & 2 & 1 & 1 & 4 & 4 & 4 & 4 & 1 & 1 & 5 & 5 & 1 & 1 & 1 \\
\hline
\end{tabular} \begin{tabular}{|l|l|l|l|l|l|l|l|l|l|l|l|l|l|l|l|l|l|l|l|l|l|l|}
\hline 1 & 1 & 2 & 2 & 2 & 2 & 1 & 1 & 2 & 2 & 1 & 1 & 4 & 4 & 4 & 4 & 1 & 1 & 5 & 5 & 1 & 1 & 1 \\
\hline
\end{tabular}

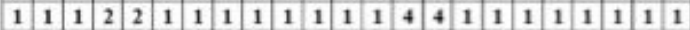

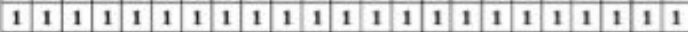
\begin{tabular}{|l|l|l|l|l|l|l|l|l|l|l|l|l|l|l|l|l|l|l|l|l|l|l|}
\hline 1 & 1 & 1 & 1 & 1 & 1 & 1 & 1 & 1 & 1 & 1 & 1 & 1 & 1 & 1 & 1 & 1 & 1 & 1 & 1 & 1 & 1 & 1 \\
\hline
\end{tabular}

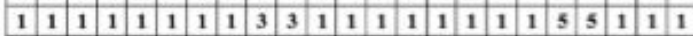
\begin{tabular}{|l|l|l|l|l|l|l|l|l|l|l|l|l|l|l|l|l|l|l|l|l|l|l|}
\hline 1 & 1 & 1 & 2 & 2 & 1 & 1 & 3 & 3 & 3 & 3 & 1 & 1 & 4 & 4 & 1 & 1 & 5 & 5 & 5 & 5 & 1 & 1 \\
\hline
\end{tabular} \begin{tabular}{|l|l|l|l|l|l|l|l|l|l|l|l|l|l|l|l|l|l|l|l|l|}
\hline 1 & 2 & 2 & 1 & 1 & 3 & 3 & 3 & 3 & 1 & 1 & 4 & 4 & 1 & 1 & 5 & 5 & 5 & 5 & 1 & 1 \\
\hline
\end{tabular}

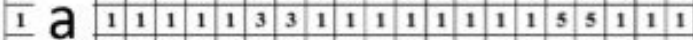
\begin{tabular}{|l|l|l|l|l|l|l|l|l|l|l|l|l|l|l|l|l|l|l|l|l|}
\hline 1 & 1 & 1 & 1 & 1 & 1 & 1 & 1 & 1 & 1 & 1 & 1 & 1 & 1 & 1 & 1 & 1 & 1 & 1 & 1 & 1 \\
\hline
\end{tabular} \begin{tabular}{|l|l|l|l|l|l|l|l|l|l|l|l|l|l|l|l|l|l|l|l|l|l|}
\hline 1 & 1 & 1 & 1 & 1 & 1 & 1 & 1 & 1 & 1 & 1 & 1 & 1 & 1 & 1 & 1 & 1 & 1 & 1 & 1 & 1 & 1 \\
\hline
\end{tabular}

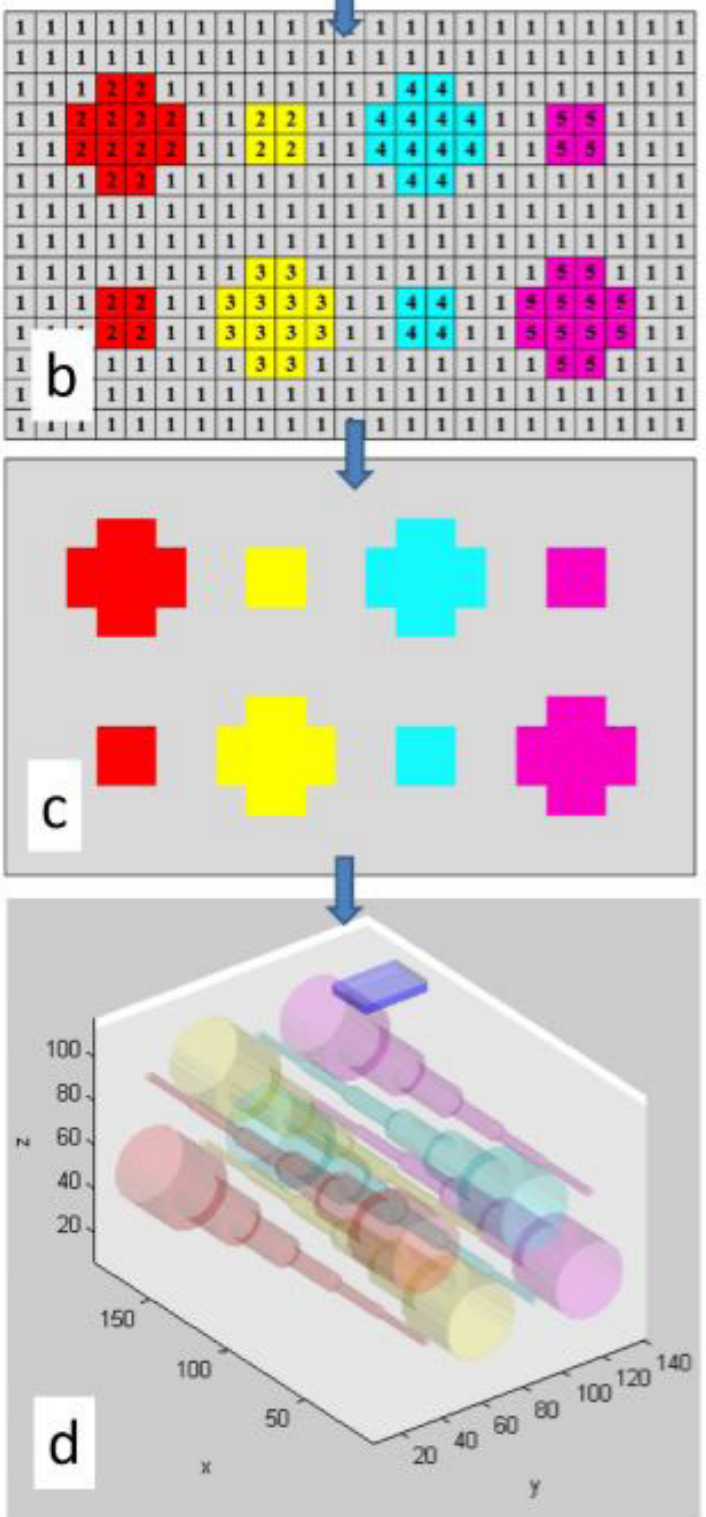

Figure 2. (a-b) Illustration of voxel values to differentiate regions, where each value stands for a different tissue. (b-c) Conversion of the values to color for better visualization. (d) depicts the rendering of the volume that simulates the phantom QA 049A from CIRS, all measures are in mm.

\section{Mesh generation}

The mesh is a combination of geometric functions to represent the anatomical and mechanical features of a domain (Kattan, 2008; Reddy, 2006; Zienkiewicz et al., 2010a; 2010b). Since our goal is to investigate the mechanical characteristics of transcutaneous biological tissue using ultrasound image, a mesh corresponding to that structure is created considering the regions and mechanical parameters set previously. Consequently, tetrahedral elements are created (Figure 3a) using the function iso2mesh - a free Matlab/Octave-based mesh generation toolbox (Fang and Boas, 2009):

$\chi=\left\{\left[P_{i}(\tau), P_{j}(\tau), P_{k}(\tau), P_{m}(\tau), \quad R(\tau)\right] \mid \tau=1, \ldots, N\right\}$

where $\tau$ is a tetrahedron element, $P_{i}(\tau), P_{j}(\tau), P_{k}(\tau)$ and $P_{m}(\tau)$ are the node indices of the corresponding element, $R(\tau)$ is the element homogeneous region, which contains the mechanical properties, and $N$ is the total number of elements of the created mesh.

\section{Strain simulation}

The deformation or strain is a shape modification procedure (Figure $3 b$ ), related to body stiffness and location, besides strength of applied pressure (Kattan, 2008; Reddy, 2006; Zienkiewicz et al., 2010a; 2010b). The strain simulation uses FEM with the previously inserted mechanical properties to compute the corresponding structural displacements; hence, simulating the deformation caused in the volume according to its stiffness and applied pressure. The elastic properties are stored in the stiffness matrix $(K)$, the computed displacements are put in the displacement vector $(U)$, and the location and value of applied pressure are inserted in the force vector $(F)$ (Kattan, 2008; Reddy, 2006; Zienkiewicz et al., 2010a; 2010b). In this study, we utilized Poisson's ratio $v=0.495$; hence, the material is considered to be nearly incompressible, isotropic and homogeneous (Korte et al., 1999; Le Floc'h et al., 2009).

Force vector $F$ is represented by the resulting vector force obtained by the applied pressure, area and location of the transducer. Therefore, the probe force vectors $f_{\text {probe }}$ are obtained by the applied localized pressure $P$ from the transducer, and its area $A_{\text {probe }}$ (Figure 4). Consequently, the nodes corresponding to the face where the pressure is applied receives the computed probe force vectors $f_{\text {probe }}$ :

$F($ node $)=\frac{f_{\text {probe }} \times A_{\sum \text { face }}}{A_{\text {probe }}}$

in which $F$ (node) is the force at the node (Figure 4), $A_{\text {probe }}$ is the area of the surface of the transducer in contact with the tissue and $A_{\sum \text { face }}$ is the sum of the areas of the faces to which the node belongs and are in the interface tissue/transducer. 
Table 1. Parameters used to simulate the phantom QA 049A from CIRS.

\begin{tabular}{lccccc}
\hline \multicolumn{1}{c}{ Parameter } & Background & $\begin{array}{c}\text { Type I } \\
\text { (red) }\end{array}$ & $\begin{array}{c}\text { Type II } \\
\text { (yellow) }\end{array}$ & $\begin{array}{c}\text { Type III } \\
\text { (blue) }\end{array}$ & $\begin{array}{c}\text { Type IV } \\
\text { (pink) }\end{array}$ \\
\hline Young Modulus $(\mathrm{kPa})$ & 25 & 8 & 14 & 45 & 80 \\
Scattering Amplitude & 5 & 1 & 1 & 1 & 1 \\
Scatterers Density (units/mm ${ }^{3}$ ) & 3 & 3 & 3 & 3 & 3 \\
Attenuation $(\mathrm{dB} / \mathrm{MHz} / \mathrm{cm})$ & 0 & 0 & 0 & 0 & 0 \\
Diameter of the insonified Cylinder $(\mathrm{mm})$ & - & 10.4 & 10.4 & 10.4 & 10.4 \\
Distance between insonified cylinder and phantom surface $(\mathrm{mm})$ & - & 30 & 30 & 30 & 30 \\
\hline
\end{tabular}

Table 2. Transducer specifications used during deformation and ultrasound simulation.

\begin{tabular}{lcc}
\hline \multicolumn{1}{c}{ Parameter } & Unit & Value \\
\hline Number of applied forces (frames) & $\mathrm{kgf}$ & 2 \\
Applied Forces & $\mu \mathrm{m} /$ Voxel & 1.5 \\
Phantom Resolution & $\mathrm{mm}$ & 1000 \\
Transducer Width & $\mathrm{mm}$ & 30 \\
Transducer Height & & 20 \\
Number of elements & & 64 \\
Number of Active Elements during Emission & $\mu \mathrm{m}$ & 1 \\
Number of Active Elements during Reception & $\mu \mathrm{m}$ & 16 \\
Element Width & $\mu \mathrm{m}$ & 440 \\
Element Height & $\mathrm{mm}$ & 5000 \\
Distance Between adjacent Elements & & 50 \\
Distance Between Elements and Tissue & $\mathrm{MHz}$ & 3 \\
Number of Emitted Ultrasound beams & $\mathrm{MHz}$ & 75 \\
Ultrasound Center Frequency & $\mathrm{m} / \mathrm{s}$ & 3.5 \\
Ultrasound Sampling Frequency & $\mathrm{mm}$ & 100 \\
Ultrasound Speed & $\mathrm{mm}$ & 1540 \\
Geometric Focus & & 64 \\
Depth of ROI & & 90 \\
Type of emission - linear or angular & angular \\
\hline
\end{tabular}
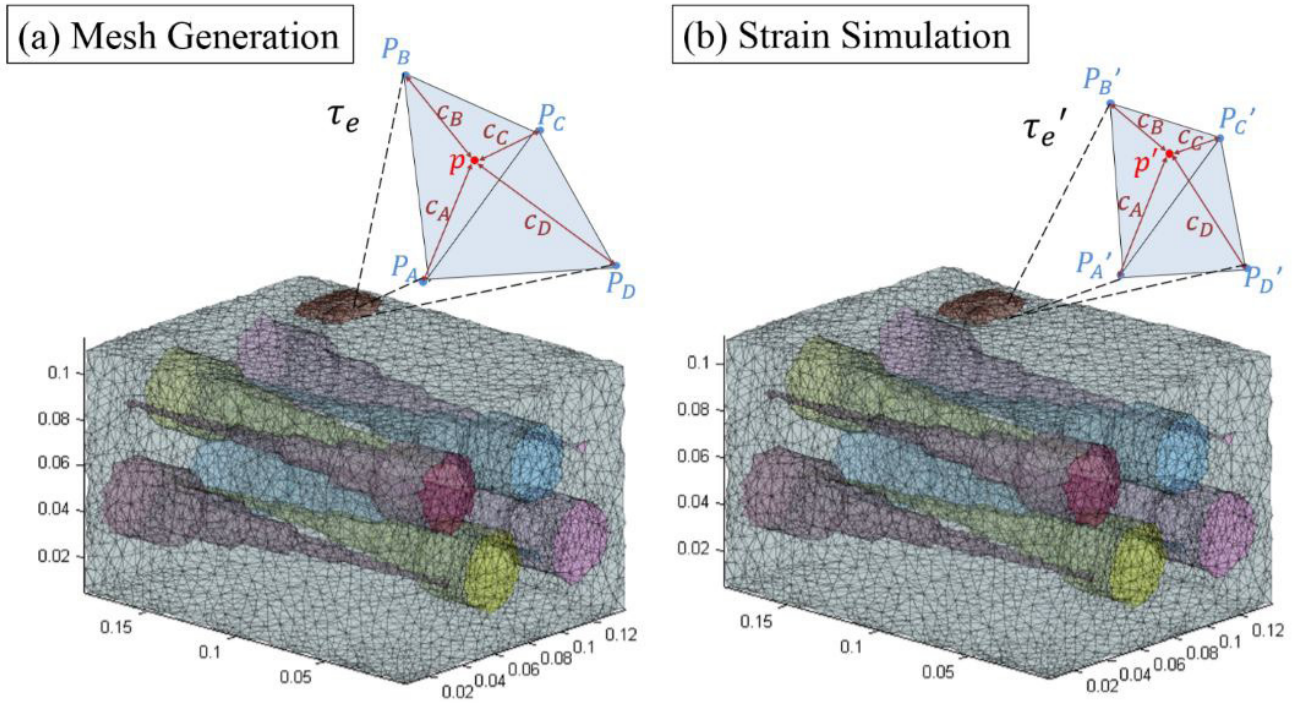

Figure 3. Volume Deformation Blocks. (a) Mesh generated with the illustration of the scatterer position $p$ inside a magnified tetrahedral element before compression. (b) Strain Simulation after the locally applied pressure and scatterer reallocation according to the tetrahedron deformation using linear isomorphism. 


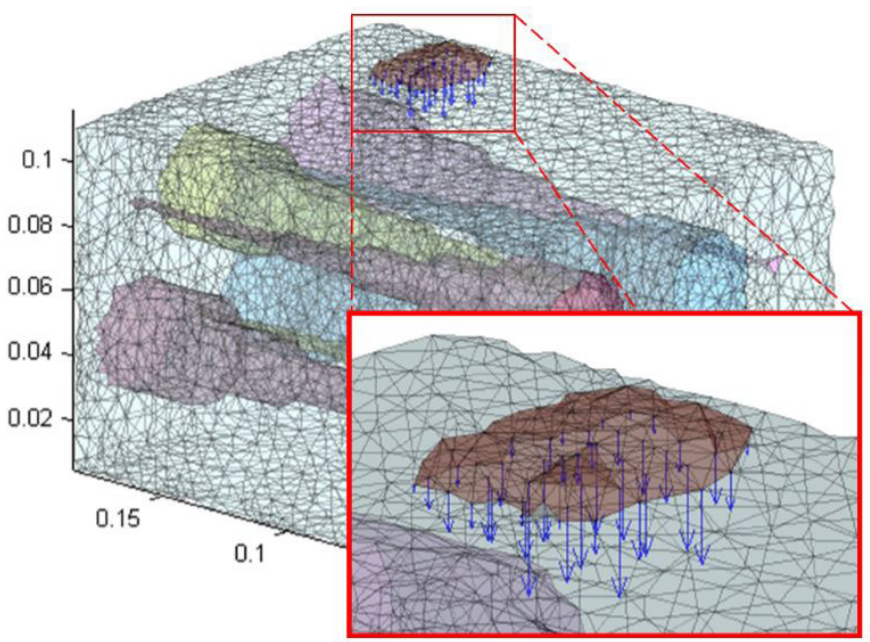

Figure 4. Close look at the transducer location. The blue arrows depict the force applied at the nodes located in the interface between the tissue and the transducer.

We considered that the box that encloses the phantom is non-deformable. Therefore, the opposite and lateral walls, in relation to the applied pressure location, are assigned as boundary conditions and they do not move during deformation.

\section{US image generation}

Realistic US Images are created from the localized volume slices by adapting Field II to the application. Field II is a program that utilizes the concept of spatial impulse responses for simulating ultrasound transducer fields and ultrasound imaging using linear acoustics (Jensen and Nikolov, 2000; Jensen and Svendsen, 1992). In order to accomplish that, the US image Generation contains four steps: Scatterers distribution, Linear isomorphism, RF simulation, and B-mode ultrasound image construction (Culjat et al., 2010).

\section{Scatterers distribution}

The scatterers are uniformly distributed inside each volumetric FEM element according to the regional acoustic parameters inserted during the Numerical Modelling section. Therefore, first, the number of scatterers of each element is calculated as follows:

$N_{\text {elem }}=V_{\text {elem }} \cdot D_{R}$

where $V_{\text {elem }}$ is the element volume and $D_{R}$ is the scatterers density of a region, to which the element belongs. Second, $N_{\text {elem }}$ are uniformly distributed inside the elements with the corresponding intensities, generating a matrix of scatterer intensities and disposition inside the volume $\left(I^{\text {scat }}\right)$, this matrix will be used to generate the non-deformed US image (I) (Figure 1c).

\section{Linear isomorphism}

Due to the deformation, the set of scatterers $\left(I^{\text {scat }}\right)$ must have their initial location modified according to the structural displacement, generating the matrix of scatterers post deformation $\left(I_{d}^{s c a t}\right)$. Therefore, in order to reproduce real image acquisitions, pre and post-deformation, morphism procedure is carried out (Figure 3).

In order to compute the corresponding matrix of scatterers for the deformed volume $\left(I_{d}^{\text {scat }}\right)$, we first associate each scatterer position from $I^{\text {scat }}$ to the element it belongs to, by computing the coordinates of the scatterer in relation to the node positions $\{P A, P B, P C, P D\}$ of its element $\tau$. Therefore, each scatterer will have a position $p=\{x, y, z\}$, determined by four constants relative to the element $\left\{c_{A}, c_{B}, c_{C}, c_{D}\right\}$ (Figure $3 a$ ); hence, $p$ is defined as:

$$
\begin{aligned}
x & =c_{A} \cdot x_{A}+c_{B} \cdot x_{B}+c_{C} \cdot x_{C}+c_{D} \cdot x_{D} \\
p=c_{A} \cdot P_{A}+c_{B} \cdot P_{B}+c_{C} \cdot P_{C}+c_{D} \cdot P_{D} \rightarrow y & =c_{A} \cdot y_{A}+c_{B} \cdot y_{B}+c_{C} \cdot y_{C}+c_{D} \cdot y_{D} \\
z & =c_{A} \cdot z_{A}+c_{B} \cdot z_{B}+c_{C} \cdot z_{C}+c_{D} \cdot z_{D}
\end{aligned}
$$

In order to ensure that the scatterer is inside the tetrahedral element, the following rules must be satisfied:

$0 \leq c_{A}, c_{B}, c_{C}, c_{D} \leq 1$

and

$c_{A}+c_{B}+c_{C}+c_{D}=1$ 
Once the tetrahedron element is deformed, the element nodes are moved to new positions $\left\{P_{a}{ }_{a}, P_{b}{ }_{b}, P_{c}{ }_{c}, P^{\prime}{ }_{d}\right\}$, hence, the scatterer position will also be reallocated $p^{\prime}=\left\{x^{\prime}, y^{\prime}, z^{\prime}\right\}$ (Figure 3b). Assuming that the transformation is linear, the constants $\left\{c_{A}, c_{B}, c_{C}, c_{D}\right\}$ will be the same after deformation. Consequently, the new scatterer position $p$ ' can be computed by knowing the new node positions after FEM deformation $\left\{P_{a}^{\prime}, P_{b}, P_{c}, P_{d}{ }_{d}\right\}$; thus (Figure 3b):

$$
p^{\prime}=c_{A} \cdot P_{A}^{\prime}+c_{B} \cdot P_{B}^{\prime}+c_{C} \cdot P_{C}^{\prime}+c_{D} \cdot P_{D}^{\prime} \rightarrow \begin{aligned}
& x^{\prime}=c_{A} \cdot x_{A}^{\prime}+c_{B} \cdot x_{B}^{\prime}+c_{C} \cdot x_{C}^{\prime}+c_{D} \cdot x_{D}^{\prime} \\
& y^{\prime}=c_{A} \cdot y_{A}^{\prime}+c_{B} \cdot y_{B}^{\prime}+c_{C} \cdot y_{C}^{\prime}+c_{D} \cdot y_{D}^{\prime} \\
& z^{\prime}=c_{A} \cdot z_{A}^{\prime}+c_{B} \cdot z_{B}^{\prime}+c_{C} \cdot z_{C}^{\prime}+c_{D} \cdot z_{D}^{\prime}
\end{aligned}
$$

By performing the morphism, the corresponding slices of the final US phantoms (Figure 1c), will reproduce realistic speckle aspects, including the dynamic of tissue movement.

\section{RF simulation}

The RF simulation is performed using Field II developed by (Jensen and Nikolov, 2000; Jensen and Svendsen, 1992). Specifically, the transducer characteristics introduced at the numerical modelling section are recalled with a sampling frequency preset to $100 \mathrm{MHz}$ to perform the ultrasound beamforming. Then, it considers the set of scatterers, $I^{\text {scat }}$, to calculate the received signal $S$. Analogously, the procedure is repeated for the case with deformation and $I^{\text {scat }}$ is utilized to obtain $S_{d}$. In order to reduce computation time, the corresponding RF signal from the $I^{\text {scat }}$ and $I_{d}^{\text {scat }}$ are generated taking into consideration only the scatterers located within a slice of the phantom with $15 \mathrm{~mm}$-thickness.

\section{B-mode ultrasound image generation}

The B-mode images (Figure 5) are created by first computing the envelope of $S$, generating $S_{e n v}$ :

$$
S_{e n v}=|S+i * H(S)|
$$

in which $H$ is the Hilbert Transform and $i$ is the imaginary unit.

The intensity of the signals related to the highly echogenic regions hinders the visualization of hypoechoic structures. Therefore, in order to reduce the dynamic range of the received signals for efficient display, compression is performed by a $\log$ function:

$$
S_{\log }=\log \left(S_{\text {env }}\right)
$$

Next, since the RF resolution is higher than the B-Mode the resulting signal is down sampled with the following sampling factor:

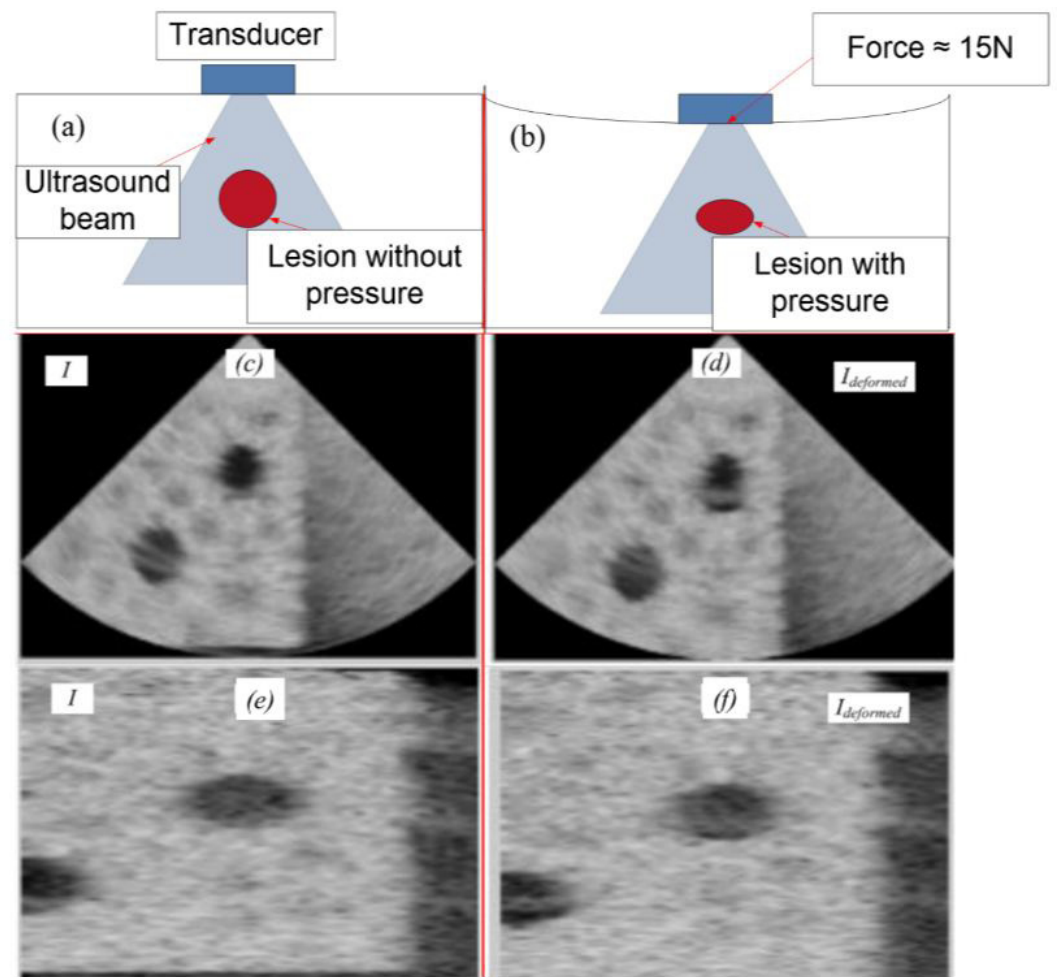

Figure 5. B-Mode Ultrasound Image Generation. (a) and (b) illustrate how the image acquisition of the physical phantom was performed without and with pressure, respectively. The final noisy images constructed with RF generated with angular (c and d) and linear (e and f) emissions for the non-deformed $I$ (c and e), and deformed $I_{\text {deformed }}(\mathrm{d}$ and $\mathrm{f}$ ) volume slice. 
$D=\frac{\operatorname{Res}_{U S} * f_{s}}{c}$

where $f_{s}$ is the sampling frequency preset to $100 \mathrm{MHz}$, $\operatorname{Res}_{U S}$ is the desired US image resolution and $c$ is the US speed. Finally, the resulting lines are distributed linearly or angularly, according to their position during acquisition, and a bilinear interpolation finishes the B-mode construction (Figure 5).

\section{Results}

The evaluation focuses on the FEM deformation accuracy, as the US images are generated by Field II, a widely used method for US image generation and corroborated in other occasions (Cardoso et al., 2012; Jensen and Nikolov, 2000; Jensen and Svendsen, 1992). Therefore, the rating was carried out by comparing the deformation obtained from the numerical phantoms created with the results from the physical phantom (CIRS Elasticity QA Phantom, Model 049 A). In order to accomplish this task, first, US images from the different "lesions" of the physical phantom were acquired without pressure and with a specific pressure. Next, a numerical volume representation of the Phantom QA Model 049A was created, and phantom images generated in the same conditions as the real acquisition. Finally, the resulting strains of the lesions investigated from the numerical and physical phantoms were compared and the correspondence between them was corroborated.

\section{US images acquisition from physical phantom}

The US data acquisitions of the physical phantom were performed using ultrasound system with the transducer (4-2MHz) (SA4-2/24). The target legions had $10.4 \mathrm{~mm}$ diameter and the center was located $30 \mathrm{~mm}$ below the phantom surface. For each lesion of the physical phantom, 10 Cross section images were acquired independently without (Figure 5a) and with pressure (Figure 5b). The force applied was approximately $15 \mathrm{~N}$, obtained by pressing the transducer with $1.5 \mathrm{~kg}$. Although this force is greater than the force used in clinical practice, it was used here to facilitate the visualization of the compression. This value was chosen taking into consideration a good trade-off between producing an identifiable strain ratio and not damaging the phantom. The transducer surface measures approximately $3 \mathrm{~cm}$ x $2 \mathrm{~cm}$.
By having the RF maps, the lesions were manually segmented and the diameter was measured in the same direction as the transducer axial direction in both uncompressed and compressed images. Then, the linear strain ratios of the lesions were computed as follows:

$\varepsilon=\frac{L-L_{o}}{L_{0}}$

where $L_{0}$ and $L$ are the lesion diameter before and after compression, respectively.

The obtained linear strain values are shown in Table 3.

\section{Numerical phantom creation}

A numerical volume representing the Phantom QA Model 049A was created (Figure 2d). The locations, dimensions and features of the box and lesions are the same as the physical phantom QA Model 049A datasheet (CIRS..., 2013). The numeric phantom properties are shown in Table 1.

The FEM deformation was performed with the same transducer dimension, locations, and applied force as the real acquisition. The boundary conditions were considered setting static the nodes located at the bottom and lateral wall of the box.

The ultrasound simulation was performed using Field II. The excitation was a two-cycle sine function and the impulse response for both emission and reception was a Hanning weighted two-cycle sine function. All parameters and values are summarized in Table 2.

A displacement map was generated using the information from the FEM deformation, and then the linear strain was calculated as follows.

$$
\varepsilon=\frac{\delta\left(p_{U}\right)-\delta\left(p_{L}\right)}{p_{U}-p_{L}}
$$

where $p_{U}$ and $p_{L}$ are the lesion upper and lowest pixel position and $\delta\left(p_{U}\right)$ is the displacement of pixel $p$.

The calculated linear strains of the numeric phantom are shown in Table 3.

The computational time used to perform the numerical modeling was $1.93 \pm 0.24 \mathrm{~s}$, while the FEM deformation consumed $114.91 \pm 13.74 \mathrm{~s}$ and the linear deformation took $14.36 \pm 2.27 \mathrm{~s}$ to be completed. The RF simulation provided by Field II had an average of $33.82 \pm 1.25 \mathrm{~s}$ per ultrasound signal.

Table 3. Strain values (\%) produced by the Physical and Numerical Phantoms.

\begin{tabular}{|c|c|c|c|c|}
\hline \multirow{2}{*}{ Phantom } & \multicolumn{4}{|c|}{ Lesions strain (\%) } \\
\hline & Type I $8 \pm 3 \mathrm{kPa}$ & Type II $14 \pm 4 \mathrm{kPa}$ & Type III $45 \pm 8 \mathrm{kPa}$ & Type IV $80 \pm 12 \mathrm{kPa}$ \\
\hline Physical & $10.1 \pm 2.1$ & $9.81 \pm 1.39$ & $4.88 \pm 1.77$ & $3.84 \pm 0.65$ \\
\hline Numerical & $11.0 \pm 3.2$ & $8.86 \pm 1.48$ & $5.69 \pm 0.81$ & $3.60 \pm 0.71$ \\
\hline
\end{tabular}


The results were obtained from a set of 10 simulations with different scatterers distribution.

As can be observed, the strain values produced by the numerical and physical phantoms are very similar. In addition, by running the $t$ student test, the null hypothesis is not rejected with significance level $\alpha=0.001$, corroborating the mechanical similarity between the two phantoms.

\section{Discussion}

A framework devoted to realistic transcutaneous phantoms was described. To our knowledge, no previous work involving numeric 3D phantom deformation, scatterers displacement and speckle noise simulation in only one platform has been published. Baldewsing et al. (2004) and Le Floc'h et al. (2009) used FEM to simulate a 2D model to predict arterial strain behavior. In Bhatti and Sridhar-Keralapura (2012) a 3D software breast phantom was built using a mechanical design tool, to investigate the biomechanics of elastography using FEM. Although the authors designed the phantom to be flexible in both, the breast geometry and biomechanical parameters, there was no further ultrasound simulation. In Maurice et al. (2004), FEM was used to calculate the strain and included the speckle noise in the images considering the acoustic impedance of each structure, with no regards to the consistency of the scatterers displacement.

Differently from the other techniques presented in the literature, we developed a method that consistently displaces the scatterers according to the results from the FEM. In other words, we consider that the scatterers map is a property of the imaged tissue that should move according to the tissue strain. The proposed approach was embedded in a Matlab Toolbox as a citationware. It may be used freely for research and educational purposes, and is found online at IVUSSim website (Universidade..., 2012). The methodology is divided into three parts: Numerical modelling, Volume deformation, US image generation. The first informs how a volume representing anatomical features may be constructed. In the second, FEM is used for the mechanical simulations of structures. Finally, in the third block, Field II and linear isomorphism are again combined (Cardoso et al., $2011 ; 2012)$ to create realistic pre-compression and post-compression US images. Future works may include different beamforming strategies, such as plane wave compounding and Fourier imaging.

The phantom visual results (Figure 5) with the deformation values corroborated with physical phantom under real acquisitions demonstrate the reliability and usefulness of the framework. Among the combination of methods to produce a unique transcutaneous phantom based on 3D deformable volumes, the major contributions of this framework is a 3D morphism procedure, in which a 3D FEM mesh is connected to the scatterers providing a novel routine of a real 3D scatterer reallocation. Consequently, realistically scatterers shifting due to regional deformation are reproduced by the generated phantoms. Since Field II (Jensen and Nikolov, 2000; Jensen and Svendsen, 1992) is a widely used and established technique, the evaluation focused on the 3D FEM accuracy, corroborated by comparing the strain results (Table 3) from numerical and physical phantoms under the same condition and similar parameters. Additionally, the student $t$ test demonstrated that the null hypotheses of both strain results, numerical and physical, is not rejected with confidence above $99.9 \%$ for all tissues.

\section{Acknowledgements}

This work was financially supported by FAPESP (process number 2011/01314-3 and 2012/15721-2) and experiments with the physical phantoms had the cooperation of the Center of Biomedical Engineering of the State University of Campinas, Brazil.

\section{References}

Anton H, Rorres C. Elementary linear algebra: applications version. 9th ed. New York: John Wiley \& Sons, Inc.; 2005

Baldewsing RA, de Korte CL, Schaar JA, Mastik F, van der Steen AFW. A finite element model for performing intravascular ultrasound elastography of human atherosclerotic coronary arteries. Ultrasound in Medicine \& Biology. 2004; 30(6):803-13. PMid:15219960. http://dx.doi.org/10.1016/j. ultrasmedbio.2004.04.005.

Bhatti SN, Sridhar-Keralapura M. A novel breast software phantom for biomechanical modeling of elastography. Medical Physics. 2012; 39(4):1748-68. PMid:22482599. http://dx.doi. org/10.1118/1.3690467.

Browne JE, Ramnarine KV, Watson AJ, Hoskins PR. Assessment of the acoustic properties of common tissue-mimicking test phantoms. Ultrasound in Medicine \& Biology. 2003; 29(7):1053-60. PMid:12878252. http://dx.doi.org/10.1016/ S0301-5629(03)00053-X.

Bude RO, Adler RS. An easily made, low-cost, tissue-like ultrasound phantom material. Journal of Clinical Ultrasound. 1995; 23(4):271-3. PMid:7797668. http://dx.doi.org/10.1002/ jcu. 1870230413 .

Cardoso FM, Moraes MC, Furuie SS. A framework to create realistic IVUS phantoms for different intraluminal pressures. In: Proceedings of the 2011 Computing in Cardiology; 2011 Sept 18-21; Hangzhou, China. New York: IEEE; 2011. p. 333-6.

Cardoso FM, Moraes MC, Furuie SS. Realistic IVUS image generation in different intraluminal pressures. Ultrasound in Medicine \& Biology. 2012; 38(12):2104-19. PMid:23062368. http://dx.doi.org/10.1016/j.ultrasmedbio.2012.08.005. 
Céspedes EI, Ophir J, Ponnekanti H, Maklad N. Elastography: elasticity imaging using ultrasound with application to muscle and breast in vivo. Ultrasonic Imaging. 1993; 15(2):73-88. PMid:8346612. http://dx.doi.org/10.1177/016173469301500201.

CIRS Tissue Simulation \& Phantom Technology. CIRS elasticity QA phantom datasheet: model 049 \& 049A [Internet]. Virginia: CIRS Tissue Simulation \& Phantom Technology; 2013. [cited 2017 Jan 05]. Available from: http://www.cirsinc.com/products/ all/74/elasticity-qa-phantoms/?details=specs

Culjat MO, Goldenberg D, Tewari P, Singh RS. A review of tissue substitutes for ultrasound imaging. Ultrasound in Medicine \& Biology. 2010; 36(6):861-73. PMid:20510184. http://dx.doi.org/10.1016/j.ultrasmedbio.2010.02.012.

Doyley MM. Model-based elastography: a survey of approaches to the inverse elasticity problem. Physics in Medicine and Biology. 2012; 57(3):R35-73. PMid:22222839. http://dx.doi. org/10.1088/0031-9155/57/3/R35.

Fang Q, Boas DA. Tetrahedral mesh generation from volumetric binary and grayscale images. In: Proceedings of the 2009 IEEE Biomedical Imaging Symposium: From Nano to Macro; 2009 June 28-July 1; Boston, MA, USA. New York: IEEE; 2009. p. 1142-5.

Hoskins PR. Simulation and validation of arterial ultrasound imaging and blood flow. Ultrasound in Medicine \& Biology. 2008; 34(5):693-717. PMid:18329162. http://dx.doi.org/10.1016/j. ultrasmedbio.2007.10.017.

Jensen JA, Nikolov SI. Fast simulation of ultrasound images. In: Proceedings of the 2000 IEEE Ultrasonics Symposium; 2000 Oct 22-25; San Juan, USA. New York: IEEE; 2000. v. 2. p. 1721-4.

Jensen JA, Svendsen NB. Calculation of pressure fields from arbitrarily shaped, apodized, and excited ultrasound transducers. IEEE Transactions on Ultrasonics, Ferroelectrics, and Frequency Control. 1992; 39(2):262-7. PMid:18263145. http://dx.doi.org/10.1109/58.139123.

Kallel F, Ophir J. A least-squares strain estimator for elastography. Ultrasonic Imaging. 1997; 19(3):195-208. PMid:9447668. http://dx.doi.org/10.1177/016173469701900303.

Kattan PI. MATLAB guide to finite elements: an interactive approach. 2nd ed. New York: Springer Berlin Heidelberg; 2008.

King DM, Moran CM, McNamara JD, Fagan AJ, Browne JE. Development of a vessel-mimicking material for use in anatomically realistic Doppler flow phantoms. Ultrasound in Medicine \& Biology. 2011; 37(5):813-26. PMid:21497719. http://dx.doi.org/10.1016/j.ultrasmedbio.2011.02.012.

Korte CL, Céspedes EI, van der Steen AFW. Influence of catheter position on estimated strain in intravascular elastography. IEEE Transactions on Ultrasonics, Ferroelectrics, and Frequency Control. 1999; 46(3):616-25. PMid:18238462. http://dx.doi. org/10.1109/58.764848.

Kurnaz MN, Dokur Z, Ölmez T. An incremental neural network for tissue segmentation in ultrasound images. Computer
Methods and Programs in Biomedicine. 2007; 85(3):187-95. PMid:17275135. http://dx.doi.org/10.1016/j.cmpb.2006.10.010.

Latifoğlu F. A novel approach to speckle noise filtering based on artificial bee colony algorithm: an ultrasound image application. Computer Methods and Programs in Biomedicine. 2013; 111(3):561-9. PMid:23806680. http://dx.doi.org/10.1016/j. cmpb.2013.05.009.

Le Floc'h S, Ohayon J, Tracqui P, Finet G, Gharib AM, Maurice RL, Cloutier G, Pettigrew RI. Vulnerable atherosclerotic plaque elasticity reconstruction based on a segmentation-driven optimization procedure using strain measurements: Theoretical framework. IEEE Transactions on Medical Imaging. 2009; 28(7):1126-37. PMid:19164080. http://dx.doi.org/10.1109/ TMI.2009.2012852.

Liang Y, Zhu H, Friedman MH. Estimation of the transverse strain tensor in the arterial wall using IVUS image registration. Ultrasound in Medicine \& Biology. 2008; 34(11):1832-45. PMid: 18620800. http://dx.doi.org/10.1016/j.ultrasmedbio.2008.04.005.

Loree HM, Tobias BJ, Gibson LJ, Kamm RD, Small DM, Lee RT. Mechanical properties of model atherosclerotic lesion lipid pools. Arteriosclerosis, Thrombosis, and Vascular Biology. 1994; 14(2):230-4. PMid:8305413. http://dx.doi. org/10.1161/01.ATV.14.2.230.

Maurice RL, Ohayon J, Finet G, Cloutier G. Adapting the lagrangian speckle model estimator for endovascular elastography: Theory and validation with simulated radiofrequency data. The Journal of the Acoustical Society of America. 2004; 116(2):1276-86. PMid:15376693. http://dx.doi. org/10.1121/1.1771615.

Ophir J, Céspedes EI, Garra B, Ponnekanti H, Huang Y, Maklad N. Elastography: ultrasonic imaging of tissue strain and elastic modulus in vivo. European Journal of Ultrasound. 1996; 3(1):49-70. http://dx.doi.org/10.1016/0929-8266(95)00134-4.

Ragazzoni F, Deandrea M, Mormile A, Ramunni MJ, Garino F, Magliona G, Motta M, Torchio B, Garberoglio R, Limone P. High diagnostic accuracy and interobserver reliability of real-time elastography in the evaluation of thyroid nodules. Ultrasound in Medicine \& Biology. 2012; 38(7):1154-62. PMid:22542262. http://dx.doi.org/10.1016/j.ultrasmedbio.2012.02.025.

Reddy JN. An introduction to the finite element method. 3rd ed. New York: McGraw-Hill; 2006.

Universidade de São Paulo - USP. Escola Politécnica. Laboratório de Engenharia Biomédica. IVUSSim website [Internet]. São Paulo: Escola Politécnica, USP; 2012. [cited 2017 Jan 05]. Available from: http://www.leb.usp.br/UltraSSim

Zienkiewicz OC, Taylor RL, Zhu JZ. The finite element method: its basis \& fundamentals. 6th ed. Great Britain: Elsevier; 2010a.

Zienkiewicz OC, Taylor RL, Zhu JZ. The finite element method: for solid and structural mechanics. 6th ed. Great Britain: Elsevier; 2010b. 


\section{Erratum}

In the article "Realistic deformable 3D numeric phantom for transcutaneous ultrasound", DOI http://dx.doi. org/10.1590/2446-4740.05616, published in Research on Biomedical Engineering, vol. $33 \mathrm{n}$. 1, on authors affiliation:

Where it reads:

\section{Fernando Mitsuyama Cardoso ${ }^{1 *}$}

Matheus Cardoso Moraes ${ }^{1}$

Sergio Shiguemi Furuie ${ }^{1}$

${ }^{1}$ School of Engineering, University of São Paulo, São Paulo, SP, Brazil.

It should be read:

\section{Fernando Mitsuyama Cardoso ${ }^{1 *}$}

Matheus Cardoso Moraes ${ }^{2}$

\section{Sergio Shiguemi Furuie ${ }^{1}$}

${ }^{1}$ School of Engineering, University of São Paulo, São Paulo, SP, Brazil.

${ }^{2}$ Institute of Science and Technology, Federal University of São Paulo, São José dos Campos, SP, Brazil. 